\begin{tabular}{|c|l|}
\hline Title & $\begin{array}{l}\text { Effect of delayed nonlinear response on femtosecond optical pulse compression by use of an organic crystal-cored fiber } \\
\text { in the normal dispersion region }\end{array}$ \\
\hline Author(s) & Morita, Ryuji; Y amashita, Mikio \\
\hline Citation & $\begin{array}{l}\text { Optics Letters, 19(18), 1459-1461 } \\
\text { https:/doi.org/10.1364/0L.19.001459 }\end{array}$ \\
\hline Issue Date & 199409-15 \\
\hline Doc URL & http://hdl.handle.net/2115/45325 \\
\hline Rights & ○ 1994 Optical Society of A merica \\
\hline Type & article \\
\hline File Information & OL19-18_1459-1461.pdf \\
\hline
\end{tabular}

Instructions for use 


\title{
Effect of delayed nonlinear response on femtosecond optical pulse compression by use of an organic crystal-cored fiber in the normal dispersion region
}

\author{
Ryuji Morita and Mikio Yamashita \\ Department of Engineering Science, Hokkaido University, Kita-13, Nishi-8, Kita-ku, Sapporo 060, Japan
}

Received March 22, 1994

\begin{abstract}
We investigate the effect of delayed nonlinear response time on pulse compression, using an organic crystal-cored fiber in the normal dispersion region. With up to third-order phase compensation, a 100-W, 100-fs hyperbolicsecant pulse is compressed to $\sim 10 \mathrm{fs}$ with a delayed nonlinear response time as well as without it. It is shown that third-order phase adjustment can compensate for the phase asymmetry in the frequency domain that is induced by the effect of the delayed nonlinear response.
\end{abstract}

Organic materials are attractive for potential applications in electro-optical switching and frequency conversion because of their large optical nonlinearities. Organic materials are also used for optical pulse compression. For example, efficient compression of femtosecond laser pulses by use of a fiber cored with the organic material 4-( $N, N$-dimethylamino)-3acetamidonitrobenzene (DAN) was demonstrated. ${ }^{1}$

Most analyses of pulse compression in the normal dispersion regime are performed without taking into account the delayed nonlinear response, whereas in the soliton-effect compression regime this delayed response is taken into account. ${ }^{2,3}$ This is because fused-silica fibers are usually utilized for pulse compression, and in many cases their response time, which was evaluated to be $2-4 \mathrm{fs},{ }^{4}$ is much shorter than the input pulse widths. However, when pulse widths are comparable with the response time the nonlinear response time becomes significant.

From our recent experiment on nonlinear femtosecond optical pulse propagation the response time of DAN was evaluated to be several tens of femtoseconds, ${ }^{5}$ causing us to consider the effect of the response time for femtosecond optical pulse compression with a DAN fiber.

In this Letter we investigate numerically the effect of the delayed nonlinear response time on pulse compression, using a DAN fiber in the normal dispersion region, as well as those of second- and third-order dispersion, self-steepening, initial frequency chirping, and propagation loss.

The modified nonlinear Schrödinger equation that we use here is

$$
\begin{aligned}
\frac{\partial u}{\partial \xi}+\Gamma u & +\frac{i}{2} \frac{\partial^{2} u}{\partial \tau^{2}}-\delta \frac{\partial^{3} u}{\partial \tau^{3}} \\
& =\frac{i}{\tau_{R}}\left\{u J\left(\tau ; \tau_{R}\right)+i s \frac{\partial}{\partial \tau}\left[u J\left(\tau ; \tau_{R}\right)\right]\right\}, \\
J\left(\tau ; \tau_{R}\right) & \equiv \int_{0}^{\infty} \mathrm{d} \tau^{\prime} \exp \left(-\tau^{\prime} / \tau_{R}\right)\left|u\left(\tau-\tau^{\prime}\right)\right|^{2},
\end{aligned}
$$

where $u$ is the normalized complex amplitude of the pulse envelope ${ }^{6}$ and

$$
\begin{array}{rlrl}
\xi & =\left|\beta_{2}\right| z / T_{0}{ }^{2}, & \tau & =\left(t-z / v_{g}\right) / T_{0}, \\
\delta & =\beta_{3} / 6\left|\beta_{2}\right| T_{0}, & s & =2 / \omega_{0} T_{0}, \\
\Gamma & =\alpha L_{D} / 2, & \tau_{R}=T_{R} / T_{0}, \\
L_{D} & =T_{0}{ }^{2} /\left|\beta_{2}\right| & \left(\beta_{2}>0\right) .
\end{array}
$$

$z$ is the distance along the fiber, $\tau$ is the normalized retarded time measured in a frame of reference moving along the fiber at the group velocity $v_{g}, \omega_{0}$ is the central frequency, $T_{0}$ is the initial pulse-width parameter [FWHM $T_{p}=2 \ln (1+\sqrt{2}) T_{0} \simeq 1.763 T_{0}$ ], $\alpha$ is the propagation loss, and $\beta_{2}$ and $\beta_{3}$ are the second- and third-order dispersions, respectively. The nonlinear refractive index $n_{2}$ is included in the definition of the normalized amplitude $u$. Here the response function is assumed to be an exponential form specified by a response time $T_{R}$ in Eq. (1b), and the instantaneousresponse part of the nonlinear refractive index is assumed to be negligible.

If $T_{R} \ll T_{p}$ (i.e., $\tau_{R} \ll \tau_{p}=T_{p} / T_{0}$ ), then $J\left(\tau ; \tau_{R}\right)$ can be approximated by the form of the Taylor expansion, and the right-hand side of Eq. (1a) becomes

$$
\begin{aligned}
& i\left\{u(\tau)\left[|u(\tau)|^{2}-\tau_{R} \frac{\partial}{\partial \tau}|u(\tau)|^{2}+\tau_{R}{ }^{2} \frac{\partial^{2}}{\partial \tau^{2}}|u(\tau)|^{2}-\ldots\right]\right. \\
& \left.\quad+i s \frac{\partial}{\partial \tau}\left[u(\tau)|u(\tau)|^{2}-\tau_{R} u(\tau) \frac{\partial}{\partial \tau}|u(\tau)|^{2}+\ldots\right]\right\}, \text { (2) }
\end{aligned}
$$

where the first term represents the self-phase modulation, the second term represents the self-frequency shift, and the fourth term represents the selfsteepening. This approximated form is usually applied in most analyses of nonlinear pulse propagation; however, this approximation is no longer valid when $T_{R} \sim T_{p}$ or $T_{R}>T_{p}$. Therefore we use Eqs. (1a) and (1b) in our calculation of a DAN fiber, which has a large delayed response time.

The fiber input pulses are assumed to have an amplitude shape given by

$$
u(\xi=0, \tau)=\operatorname{sech}(\tau) \exp \left(-i C \tau^{2} / 2\right),
$$

where $C$ is the parameter representing initial linear frequency chirp. 
Table 1. Dependence of the Compressed Pulse Width $T_{p, c}$ and the Optimum

Propagation Length $z_{\text {opt }}$ on the Response Time $T_{R}$ and the Chirp Parameter $C$

\begin{tabular}{|c|c|c|c|c|c|c|c|c|}
\hline \multirow[b]{3}{*}{$C$} & \multirow{2}{*}{\multicolumn{4}{|c|}{$\begin{array}{l}\text { Compressed Pulse } \\
\text { Width } T_{p, c}(\mathrm{fs}) \\
\text { esponse Time } T_{R}(\mathrm{fs})\end{array}$}} & \multirow{2}{*}{\multicolumn{4}{|c|}{$\begin{array}{l}\text { Optimum Propagation } \\
\text { Length } z_{\text {opt }}(\mathrm{mm}) \\
\text { Response Time } T_{R}(\mathrm{fs})\end{array}$}} \\
\hline & & & & & & & & \\
\hline & 0 & 10 & 20 & 30 & 0 & 10 & 20 & 30 \\
\hline$\Delta$ & 12.1 & 10.0 & 10.3 & 10.4 & 92 & 0.460 & 0. & 0.40 \\
\hline-1 & 11.5 & 10.9 & 10.9 & 9.54 & 0.493 & 0.487 & 0.474 & 0.454 \\
\hline-0.4 & 11.3 & 10.5 & 9.77 & 10.4 & 0.516 & 0.493 & 0.431 & 0.426 \\
\hline 0 & 11.1 & 9.85 & 10.5 & 11.2 & 0.460 & 0.572 & 0.401 & 0.421 \\
\hline+0.4 & 10.9 & 10.8 & 11.0 & 8.99 & 0.447 & 0.379 & 0.416 & 0.516 \\
\hline+1 & 10.6 & 11.2 & 9.13 & 9.72 & 0.454 & 0.368 & 0.506 & 0.500 \\
\hline+2 & 9.92 & 9.85 & 9.17 & 8.67 & 0.546 & 0.513 & 0.473 & 0.651 \\
\hline
\end{tabular}

The parameters that we used for a DAN crystalcored fiber are central wavelength $\lambda_{0}=630 \mathrm{~nm}$, core diameter $=2.5 \mu \mathrm{m}, n_{2}=2.1 \times 10^{-18} \mathrm{~m}^{2} / \mathrm{V}^{2},{ }^{7} \beta_{2}=$ $1.85 \times 10^{-24} \mathrm{~s}^{2} / \mathrm{m}, \beta_{3}=3.79 \times 10^{-39} \mathrm{~s}^{3} / \mathrm{m},{ }^{7,8} \alpha=$ $14 \mathrm{~dB} / \mathrm{cm}\left(\delta=6.0 \times 10^{-3}, \Gamma=2.8 \times 10^{-1}\right)$, and input pulse width $T_{p \text {,in }}=100 \mathrm{fs}\left(T_{0}=56.7 \mathrm{fs}\right)$.

After solving relations (1) by using the split-step Fourier method, ${ }^{9}$ we carry out the calculation of up to third-order phase compensation for output pulses from fibers to obtain compressed pulses. That is, we determine the compensated phase $\phi_{c}$ in the frequency domain

$$
\phi_{c}=\phi(\omega)-\left[a_{2}\left(\omega-\omega_{0}\right)^{2}+a_{3}\left(\omega-\omega_{0}\right)^{3}\right]
$$

where $\phi(\omega)$ is the precompensated phase of the fiber output pulses, so that the compensated pulse widths are minimized by the adjustment of parameters $a_{2}$ and $a_{3}$. This adjustment is usually performed with prism and grating pairs in a practical experiment.

Table 1 shows the dependence of the compressed pulse with $T_{p, c}$ and the optimum propagation length $z_{\text {opt }}$ on the response time $T_{R}$ for the DAN crystal-cored fiber (the input peak power $P_{0}=100 \mathrm{~W}$, the input pulse width $T_{p \text {,in }}=100 \mathrm{fs}$, and the chirp coefficient $C=0, \pm 0.4, \pm 1, \pm 2)$. It is found that, even with the delayed response, a 100-W 100-fs hyperbolic-secant pulse is compressed to $\sim 10 \mathrm{fs}$. The same compression is also seen without the delayed response, in the range of $|C| \leq 2$. As Table 1 shows, the optimum propagation lengths $z_{\text {opt }}$ are $\sim 0.5 \mathrm{~mm}$, for which the propagation loss is low enough.

Figure 1(a) shows output spectra from the fiber at $T_{R}=0$ and $30 \mathrm{fs}$, along with an input spectrum. The blue-side broadening in the spectrum that is due to self-steepening is clearly seen at $T_{R}=0 \mathrm{fs}$, whereas the frequency down shift (corresponding to the red shift) occurs as a result of the delayed response effect and suppresses the blue-side broadening at $T_{R}=30 \mathrm{fs}$. In addition, the effect of third-order dispersion may result in the spectral asymmetry for both $T_{R}$ values. Figure 1(b) shows one of the typical compressed pulse profiles $\left(T_{R}=30 \mathrm{fs}\right)$ with fiber input and output pulses $\left(T_{R}=0\right.$ and $\left.30 \mathrm{fs}\right)$ in the time domain. The precompensated pulse at $T_{R}=30 \mathrm{fs}$ has a small peak in the leading edge as a result of the delayed response. This behavior is explained as follows: since the red components travel faster than the blue components in the normal dispersion regime, the red shift that is due to the delayed response corresponds to the forming of a small peak in the leading edge in the time domain. The compressed pulse shape is slightly asymmetric, and the compressed pulse quality $\bar{Q}_{c}$ (the ratio of compressed pulse energy to precompensated energy) is $\sim 0.73$.

Figure 2 depicts the precompensated phase $\phi(\omega)$ of the fiber output pulse in the frequency domain at $C=0$ with or without the delayed response $\left(T_{R}=0\right.$, $10,20$, and $30 \mathrm{fs})$. It is clearly seen that the shape of $\phi(\omega)$ becomes more asymmetric with increasing $T_{R}$. Therefore efficient pulse compression with the delayed response requires third-order phase compensation. By sufficient adjustment of third-order phase compensation, pulses are efficiently compressed with the delayed response, as shown in Table 1 , confirm-
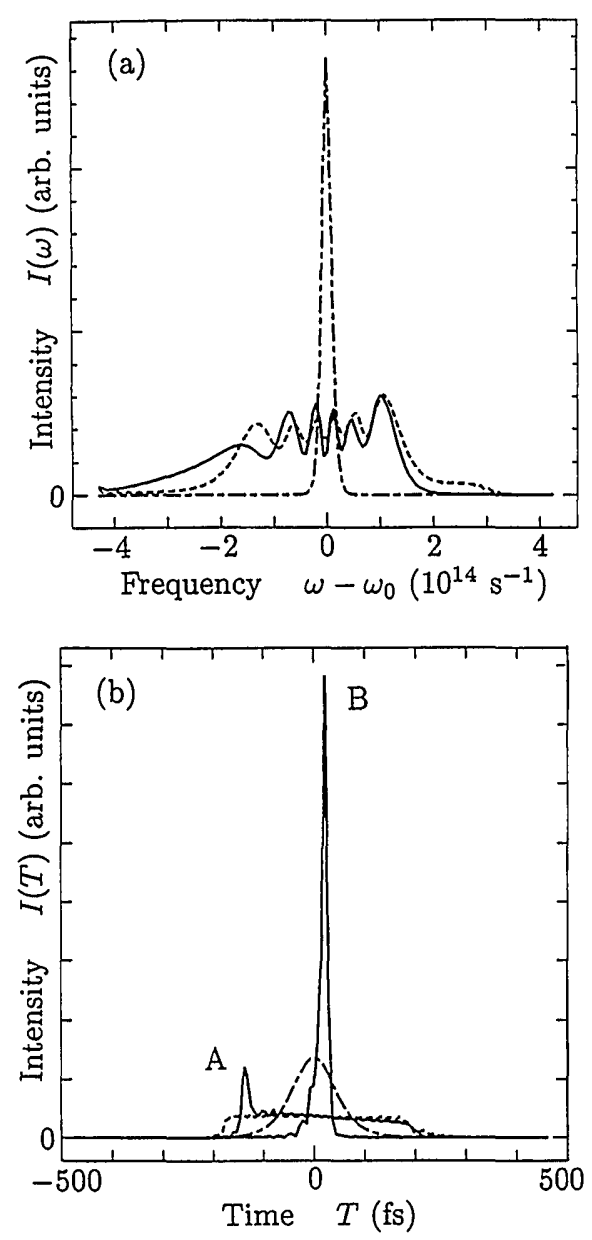

Fig. 1. (a) Typical fiber output spectra for response time $T_{R}=30 \mathrm{fs}$ (solid curve) and $T_{R}=0 \mathrm{fs}$ (dashed curve) at the distance $z=0.421 \mathrm{~mm}$. The dotted-dashed curve represents the spectrum for the input pulse (input power $P_{0}=100 \mathrm{~W}$, pulse width $T_{p \text {,in }}=100 \mathrm{fs}$, and chirp parameter $C=0$ ). (b) Typical fiber output pulses for $T_{R}=30 \mathrm{fs}$ (solid curves: A, the precompensated pulse; B, the compensated pulse) and $T_{R}=0 \mathrm{fs}$ (dashed curve, the precompensated pulse) at $z=0.421 \mathrm{~mm}$. The dotted-dashed curve represents the input pulse. $T$ is the time in the coordinate system moving at the average group velocity of the pulse. 


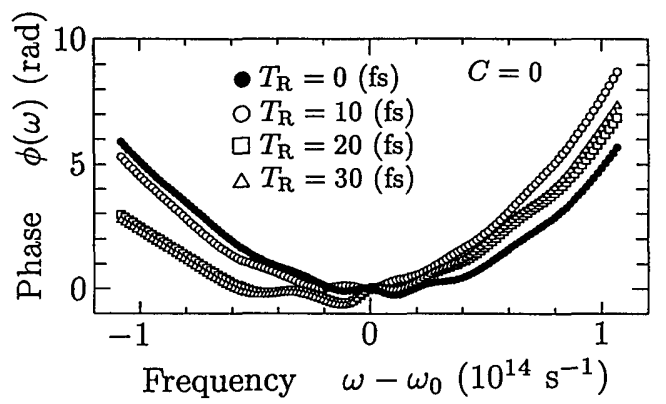

Fig. 2. Fiber output phase $\phi(\omega)$ in the frequency domain at the optimum propagation lengths $z_{\text {opt }}$. The parameters are $P_{0}=100 \mathrm{~W}, T_{p, \text { in }}=100 \mathrm{fs}$, and $C=0$.
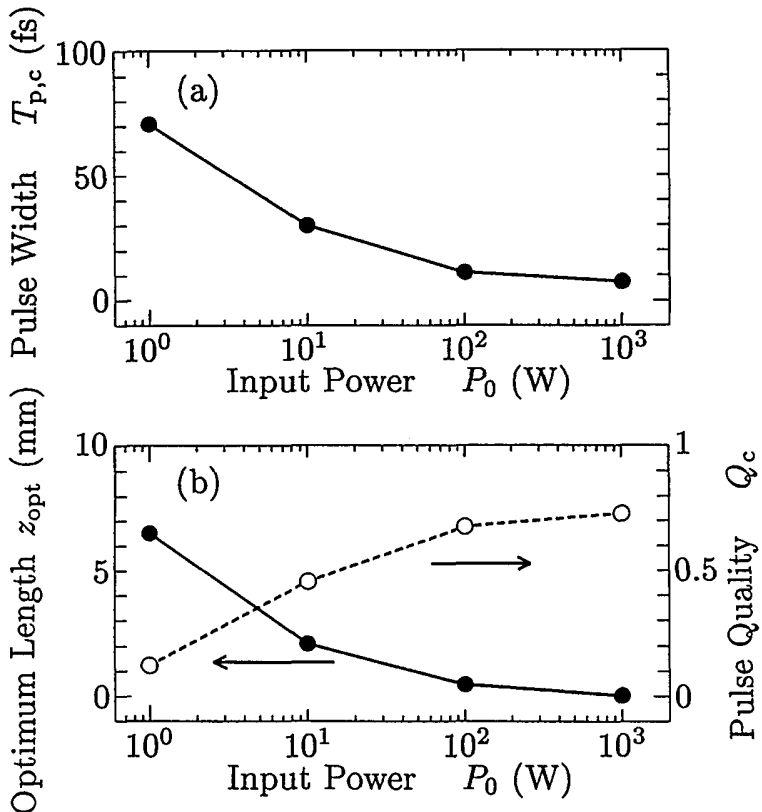

Fig. 3. Dependence of (a) the compressed pulse width $T_{p, c}$ and (b) the optimum propagation length $z_{\text {opt }}$ (filled circles) and the pulse quality $Q_{c}$ (open circles) on the input power $P_{0}$. The parameters are $T_{p, \text { in }}=100 \mathrm{fs}, T_{R}=0 \mathrm{fs}$, and $C=0$.

ing that third-order phase compensation is essential for efficient pulse compression with the delayed response. It was believed that the delayed response degrades pulse compression. This is because only second-order phase compensation has been taken into account so far. Third-order phase adjustment accompanied by second-order compensation is efficient for pulse compression when a delayed response time is involved.

At $T_{R}=0 \mathrm{fs}$ there is an enhancement of compression with positive initial chirp and a degradation of compression with negative initial chirp, as shown in Table 1. This is also seen in soliton-effect compression $^{10}$; however, it is not generalized when $T_{R} \neq 0$.

Next, let us consider the effect of the propagation loss. Figure 3 shows the dependence of the compressed pulse width $T_{p, c}$, the optimum fiber length $z_{\text {opt }}$, and the compressed pulse-quality factor $Q_{c}$ (the ratio of compressed pulse energy to input pulse en- ergy, including the effect of propagation loss) on the input power $P_{0}$ when $C=0$ and $T_{R}=0$. It is found that $z_{\text {opt }}$ and $T_{p, c}$ decrease with increasing $P_{0}$. On the other hand, $Q_{c}$ increases with the $P_{0}$ value. This is because the effect of the propagation loss reduces the pulse quality $Q_{c}$ as the propagation length increases. Because of the high propagation loss of $14 \mathrm{~dB} / \mathrm{cm}$ for the DAN fiber, an input power of more than $\sim 10 \mathrm{~W}$ is needed for efficient pulse compression. Therefore we require higher-quality DAN crystals for efficient pulse compression, using relatively low-power and long-pulse lasers such as laser diodes.

In contrast, high-power light sources such as a Ti:sapphire laser do not need the long propagation distance. For example, an $\sim 100-\mu \mathrm{m}$-thick DAN film, which is much easier to fabricate, may be promising for efficient pulse compression with $\sim 1 \mathrm{~kW}$ of input power.

In conclusion, we have numerically investigated effects of delayed nonlinear response and propagation loss on pulse compression, using an organic crystal-cored fiber in the normal dispersion region. By use of third-order phase compensation, even when a delayed nonlinear response time is involved, a $100-\mathrm{W} 100-\mathrm{fs}$ hyperbolic-secant pulse is compressed to $\sim 10 \mathrm{fs}$. It must be emphasized that the thirdorder phase adjustment can compensate for the phase asymmetry in the frequency domain that is induced by the effect of the delayed nonlinear response. Furthermore, a thin DAN crystal film is promising for efficient pulse compression at high power, whereas a reduction of the propagation distance by an improvement in crystal quality is needed at low power.

The authors are grateful to $H$. Sone for his help in the computations. This study was partially supported by a Grant-in-Aid for Scientific Research on Priority Areas, Ultrafast and Ultraparallel Optoelectronics from the Minstry of Education, Science and Culture of Japan.

\section{References}

1. M. Yamashita, K. Torizuka, T. Uemiya, and J. Shimada, Appl. Phys. Lett. 58, 2727 (1991).

2. P. V. Mamyshev, P. G. J. Wigley, J. Wilson, G. I. Stegeman, V. A. Semenov, E. M. Dianov, and S. I. Miroshnichenko, Phys. Rev. Lett. 71, 73 (1993).

3. S. Liu and W. Wang, Opt. Lett. 18, 1911 (1993).

4. A. B. Grudinin, E. M. Dianov, D. V. Korobkin, A. M. Prokhorov, V. N. Serkin, and D. V. Khaĭdarov, JETP Lett. 46, 221 (1988).

5. R. Morita, H. Sone, C. Ohshima, and M. Yamashita, in Conference on Lasers and Electro-Optics, Vol. 11 of 1993 OSA Technical Digest Series (Optical Society of America, Washington, D.C., 1993), p. 468.

6. See, for example, G. P. Agrawal, Nonlinear Fiber Optics (Academic, San Diego, Calif., 1989).

7. M. Yamashita, K. Torizuka, and T. Uemiya, Appl. Phys. Lett. 57, 1301 (1990).

8. P. Kerkoc, M. Zgonik, K. Sutter, Ch. Bosshard, and P. Günter, Appl. Phys. Lett. 54, 2062 (1989).

9. G. P. Agrawal, Opt. Lett. 15, 224 (1990).

10. K. C. Chan and H. F. Liu, Opt. Lett. 14, 1150 (1993). 